\section{A method of testing for serial correlation in univariate repeated-measures analyses of variance}

\author{
JOHN P. HATCH, ERWIN M. HEARNE III, \\ and GARY M. CLARK \\ University of Texas Health Sciences Center \\ San Antonio, Texas 78284
}

Many behavioral experiments utilize a two-factor split-plot design having one between-subjects grouping factor and one within-subjects repeated-measures factor. Subjects are assigned at random to the various levels of the grouping factor, but all subjects are exposed to all levels of the repeated-measures factor. Very often, the repeated-measures factor represents levels of a temporal variable (e.g., laboratory sessions or experimental trials). There are two assumptions concerning the variance-covariance matrices that must be met for a valid univariate test of the trials effect or the Trials by Groups interaction effect. First, the variance-covariance matrices for a set of orthonormal variables corresponding to the effect of the repeated factor must be equal across groups. The Box M test may be used to test this assumption (Huynh \& Mandeville, 1979). Second, the common covariance matrix must be spherical. An approximate test for this assumption appears in Huynh and Feldt (1970). ${ }^{1}$ When these assumptions are not met, the use of a multivariate test or reducing the degrees of freedom on repeated-measures factors (Geisser \& Greenhouse, 1958) to yield a conservative but more robust univariate $F$ test are recommended. The purpose of this discussion is to comment briefly on a particular covariance matrix structure that is not spherical and to introduce a method with which to further explore the covariance matrix structure when violations of the sphericity assumption occur.

When the repeated-measures factor is time, observations made closer together tend to be more highly correlated than those made further apart. One such pattern is the serial correlation pattern, and it can be a common cause of sphericity assumption violations in behavioral research. We recently examined the effects of the serial correlation pattern on conventional alpha levels (Hearne, Clark, \& Hatch, in press) and found that in extreme cases of the pattern (correlation of trial levels one step apart $=.8$ ) actual alpha levels were more than three times the nominal level. Thus, certain types of covariance matrix structure that violate the sphericity

Address requests for reprints to John P. Hatch, Department of Psychiatry, Medical School, University of Texas Health Science Center at San Antonio, 7703 Floyd Curl Drive, San Antonio, Texas 78284. E. M. Hearne III is with the Department of Surgery, Division of Otorhinolaryngology; G. M. Clark is with the Department of Medicine, Division of Oncology. assumption make the univariate $\mathrm{F}$ tests very liberal. On the other hand, when the correlation of trial levels one step apart was equal to .4 , the serial correlation pattern had little effect on Type I errors.

A maximum-likelihood ratio test statistic was developed (Hearne et al., in press; Hearne, Clark, \& Hatch, Note 1) that is used with a test for sphericity to detect the presence of the serial correlation pattern. The likelihood ratio test statistic is expressed as $\ell=L(\hat{\omega}) /$ $L(\hat{\Omega})$, where $L(\hat{\omega})$ is the likelihood evaluated at $\hat{\omega}$, the null hypothesis, and $L(\hat{\Omega})$ is the likelihood evaluated at $\hat{\Omega}$ under the general space. A recent simulation study showed that for some parameter sets, the likelihood ratio test identified the serial correlation structure, whereas the sphericity test failed to detect this nonspherical pattern (Hearne \& Clark, Note 2). Therefore, in some data sets, a clearly nonspherical matrix structure can be missed if the sphericity test is used alone. Also, by using tabled values of alpha for appropriate experimental parameters, the researcher can further explore the probable impact of the matrix structure on nominal alpha levels. Finally, investigators may be able to use the test to identify the origins of the serial correlation patterns in their data or to evaluate the effect of various experimental manipulations on the pattern.

Availability. A computer program to calculate the serial correlation test statistic is available from the authors upon request. The program is written as a subroutine in FORTRAN IV and is currently used on a DEC System 2060 computer. The use of complex numbers is required, but there are no other systemspecific restrictions. Subroutines from the International Mathematical and Statistical Libraries (IMSL) are used for matrix inversion and for the solution of a cubic equation. Raw data are input from a user-provided main program, and the value of the chi-square statistic is returned. Arrangements for transportation of the program may be made by contacting Gary $\mathbf{M}$. Clark, Division of Oncology, Department of Medicine, University of Texas Health Science Center at San Antonio, 7703 Floyd Curl Drive, San Antonio, Texas 78284.

\section{REFERENCE NOTES}

1. Hearne, E. M., Clark, G. M., \& Hatch, J. P. The effect of a certain pattern covariance structure on univariate repeated measures analysis. Paper presented at the meeting of the American Statistical Association, Detroit, Michigan, August 1981.

2. Hearne, E. M., \& Clark, G. M. Validity and power investigation of a test for serial correlation in univariate repeated measures analysis. Paper presented at the meeting of the American Statistical Association, Cincinnati, Ohio, August 1982.

\section{REFERENCES}

Geisser, S., \& Greenhouse, S. W. An extension of Box's results on the use of the $F$ distribution in multivariate analysis. Annals of Mathematical Statistics, 1958, 29, 885-891. 
Hearne, E. M., Clark, G. M., \& Hatch, J. P. A test for serial correlation in univariate repeated measures analysis. Biometrics, in press.

Huynh, H., \& Feldt, L. S. Conditions under which mean square ratios in repeated measurement designs have exact $\mathrm{F}$-distributions. Journal of the American Statistical Association, 1970, 65, 1582-1589.

HuYNh, H., \& Mandeville, G. K. Validity conditions in repeated measures designs. Psychological Bulletin, 1979, 86, 964-973.

\section{NOTE}

1. A test of the sphericity assumption may be requested in using Program BMDP2V of Biomedical Computer Programs, University of California Press, University of California, Los Angeles, 1979.

(Accepted for publication August 9, 1982.) 